\title{
Biología pesquera de la langosta Panulirus gracilis en Playa Lagarto, Guanacaste, Costa Rica
}

\author{
Helven Naranjo Madrigal \\ San Vicente, Moravia, San José, Costa Rica; helvenn@hotmail.com
}

Recibido 18-V-2010. Corregido 16-XII-2010. Aceptado 24-I-2011.

\begin{abstract}
Biology and fishery of the lobster Panulirus gracilis in Playa Lagarto, Guanacaste, Costa Rica. Panulirus gracilis is a high valuable lobster species with considerable captures along the tropical Pacific coast. In this study, I present some biological and fishery parameters described after a sample of 843 lobsters, landed in Playa Lagarto from November 2007 to October 2008. From landing records, a total of $74.9 \%$ of lobsters were below the minimum legal catch size (80mm CL). Carapace lengths were in the range of 42.8 and $143.6 \mathrm{~mm}$ for males and 115 and $35.8 \mathrm{~mm}$ for females. The size structure showed a wide overlapping of population segments, and a trend to increase with depth, where lung diving and "hooka" diving operations take place. Sex ratio was 1.36 M:H. The relationship between weight and LC revealed that females are heavier than males of the same size, and this difference was significant $(\mathrm{p}<0.05)$. The von Bertalanffy growth parameters for males and females respectively ( $\mathrm{K}=0.45-0.38$, $\mathrm{LC} \infty=166.9-121.7)$ showed accelerated growth compared to other species. Males observed a higher growth rate than females. Furthermore, natural mortality (M), total mortality (Z) and fishing mortality $(F)$ was higher in males $(0.49-2.34-1.92)$ than in females $(0.47-1.82-1.42)$. Recruitment was continuous for both sexes during the year, with an elevated intensity of $18.5 \%$ in July. Under the current fishing regime the population could be at risk of collapse, as indicated by the high exploitation rate (E) 0.80 . Rev. Biol. Trop. 59 (2): 619-633. Epub 2011 June 01.
\end{abstract}

Key words: Panulirus gracilis, fishery, Playa Lagarto, Costa Rica.

Las especies de la familia Palinuridae se encuentran distribuidas en la región tropical y templada, expuestas a una gran presión pesquera, tanto para la pesca de subsistencia como comercial. La región del Mar Caribe, el Sureste de los océanos Atlántico e Índico son los de mayor aporte a las capturas (70\%) a nivel mundial (Morgan 1980). Al mismo tiempo estas especies representan un recurso pesquero de importancia y juegan un papel destacado en las actividades socio-económicas de las comunidades costeras (Briones et al. 1997, Cruz 2002).

El sector pesquero costarricense es principalmente artesanal y la mayor actividad pesquera del país se desarrolla en las aguas del Pacífico (INCOPESCA 2001). Además, algunas pesquerías costeras de pequeña escala se encuentran asociadas a la explotación de invertebrados marinos.

Debido a su alto valor económico, la langosta espinosa verde Panulirus gracilis (Streets 1871) ha experimentado una alta presión pesquera en toda su área de distribución, que va desde Baja California hasta el sur de Perú (Holthuis 1991). Además, según el Instituto Costarricense de Pesca y Acuicultura (2001) esta especie constituye un importante recurso de relevancia económica para los pescadores artesanales en la costa pacífica de nuestro país. No obstante, la pesca en Costa Rica presenta problemas de manejo, sobreexplotación y ordenamiento. Debido a ello, una evaluación apropiada de los recursos que son objetivo de la pesca continúa siendo prioritaria, esto con 
el fin de generar estrategias adecuadas para el aprovechamiento sostenible de los mismos.

No existen antecedentes de información biológica pesquera que permita determinar el estado en que se encuentra el stock del recurso langosta en esta zona. Por dicha razón, la presente investigación está orientada al análisis de la pesquería de $P$. gracilis por medio de la evaluación de algunos parámetros poblacionales por estrato de profundidad donde se utilizan las dos modalidades de pesca.

\section{MATERIALES Y MÉTODOS}

El presente estudio se realizó en Playa Lagarto ubicada en el distrito 27 de Abril perteneciente al cantón de Santa Cruz en la provincia de Guanacaste, Costa Rica (10 $07^{\circ} .23^{\prime}$ ' N - 85 $47^{\prime} .97^{\prime \prime}$ W). Playa Lagarto posee una extensión aproximada de $800 \mathrm{~m}$, está dispuesta en forma de ensenada con arrecifes a los costados, rocas a flor de agua y sumergidas. En el sector sureste existe un pequeño estero con suave pendiente que hace escurrir el agua. No presenta fuerte oleaje, lo que facilita la movilización de las embarcaciones de este puerto artesanal.

Las modalidades de pesca tradicionales utilizadas para la captura de la langosta en esta zona y en general en la región de Santa Cruz son el buceo libre o a pulmón y el buceo con compresor. En el primero, se opera a profundidades no mayores a los $11 \mathrm{~m}$ dependiendo de la capacidad y habilidad del pescador. Mientras que en el segundo se puede acceder a zonas profundas más allá de los $30 \mathrm{~m}$.

A partir de los desembarques y de la captura obtenida por los buzos que trabajan a pulmón en Playa Lagarto, se tomó una muestra de 843 langostas; a las cuales se les medió la longitud del cefalotórax (LC) con la ayuda de un vernier marca Scala con 0.01 de precisión. Esta medida se tomó desde el reborde de las espinas supra-orbitales hasta el extremo posterior del caparazón. Cabe señalar que el muestreo de las embarcaciones fue al azar y que las tallas que se capturaron no estuvieron condicionadas por alguna regulación, ya que en la zona no existe vigilancia que permita el cumplimiento de las mismas.

Con una balanza electrónica se determinó el peso total de los ejemplares; el sexo y el estado reproductivo de las hembras y se anotó la presencia de masa espermatófora y/o huevos con base en las características externas de los ejemplares.

Para analizar datos de frecuencia de tallas, peso, edad y otros análisis estadísticos se utilizaron los programas computacionales estadísti$\cos \mathrm{R}$ y FiSAT II.

En el estudio de crecimiento de la langosta $P$. gracilis se obtuvieron los parámetros de talla asintótica del cefalotórax o la talla promedio máxima alcanzada por un individuo $\left(L_{\infty}\right)$ y la velocidad media de crecimiento en que se alcanza la talla asintótica $(K)$, según la ecuación de von Bertalanffy (1934):

$$
L_{t}=L_{\infty}\left(1-E X P\left(-K\left(t-t_{0}\right)\right)\right)
$$

donde $K$ es el coeficiente de crecimiento, $L_{\infty}$ la talla asintótica, $L_{t}$ la talla a la edad t (en años) y $t_{0}$ la edad teórica en la cual el animal tiene una talla de 0 . Para efectos de este estudio este último valor fue llevado a cero.

La curva que se obtiene de esta ecuación indica un crecimiento continuo, que no es real para los crustáceos, ya que estos organismos presentan un crecimiento escalonado propiciado por los incrementos en longitud que ocurren durante las mudas (Caddy 1987). De esta forma, el crecimiento discontinuo de los crustáceos se representa como una curva que muestra un crecimiento promedio y no la realidad escalonada de eventos periódicos de crecimiento rápido, seguido por un tiempo determinado de ausencia de crecimiento (Hearn 2004).

Con el método de Powell-Wetherall presente en una subrutina del programa FiSAT II, se estimó la longitud asintótica $\left(L^{\infty}\right)$.

$$
\left(L-L^{\prime}\right)=a+b * L^{\prime}
$$

donde:

$$
L=\left(\frac{L_{\infty}+(Z / K) L}{1+(Z / K)}\right)
$$


Esto deriva de despejar $-L$ en la ecuación de Beverton y Holt (1956) $\mathrm{Z}=\mathrm{K}(L \infty L) /\left(L-L^{\prime}\right)$.

$$
\text { Del cual } L_{\infty}=-\mathrm{a} / \mathrm{b} \text { y } Z / K=-(1+\mathrm{b}) / \mathrm{b}
$$

El coeficiente de crecimiento $(K)$ fue estimado mediante el método Shepherd en la subrutina de "búsqueda de $K$ ". La puntuación " $S$ " es definida como:

$$
S\left(s A^{2}+s B^{2}\right)^{1 / 2}
$$

donde $s A$ y $s B$ son la bondad de ajuste de las puntuaciones $S_{t z}$ obtenidas con la origen de la ecuación de bondad de ajuste de von Bertalanffy, en el tiempo $t_{z}$ llevado a 0 y 0.25 respectivamente. $S_{t z}$ es definida como:

$$
S_{t z}=\sum_{i} T_{i} \cdot \sqrt{ } N_{i}
$$

donde $N i=$ talla de frecuencia del grupo $i$,

$$
\begin{gathered}
T_{i}=D \cdot \cos 2 \pi\left(t-t_{i}\right), \\
D=\left(\sin \pi\left(\Delta_{t}\right) / \pi\left(\Delta_{t}\right)\right), \\
t=\left(\Delta_{t} / 2\right), \\
\Delta_{t}=t_{\max }-t_{\min }, \\
t_{i}=t_{z}-(1 / K) \cdot \ln \left(1-\left(L_{i} / L_{\infty}\right)\right), \\
T_{z}=1 /(2 \pi) \cdot \tan -1(s B / s A) .
\end{gathered}
$$

Basado en la relación entre $K$ y $L \infty$ se calculó el índice de crecimiento phi primo $\left(\emptyset^{\prime}\right)$ (Munro 1982) que es una constante en la ecuación siguiente y ha sido utilizada en numerosa poblaciones de crustáceos (Defeo et al. 1992).

$$
\log K=\emptyset^{\prime}-2-\log C L_{\infty} \text { (Pauly 1979). }
$$

Medir la mortalidad natural $(M)$ de una población explotada resulta muy difícil, pero existen aproximaciones basadas en observaciones empíricas, como la fórmula de Pauly (1979), que relaciona $M$ con la temperatura de las aguas. Sin embargo, Pauly advierte que esta fórmula fue derivada para peces, y no es apropiada para crustáceos.

Rickter \& Efanov (1976) citados por Gayanillo Jr. et al. 2005 sugieren una relación entre la edad a la cual el $50 \%$ de la población alcanza la madurez sexual.

Cruz et al. (1981) diseñaron una ecuación para calcular mortalidad natural $(M)$ con base en estudios experimentales de 13 especies de langosta, entre las que se incluyen especies del género Panulirus. Dicha fórmula relaciona $M$ con la longitud máxima del cefalotórax, la tasa de crecimiento y la temperatura del agua.

En este estudio se utilizó la fórmula de Cruz et al. (1981) para calcular el valor de $M$, ya que se consideró como el más apropiado para langostas:

$$
M=-0.0277-\left(0.0004 \times \mathrm{xLC}_{\infty}\right)+(0.5397 \times K)+0.0119 \mathrm{~T}\left({ }^{\circ} \mathrm{C}\right) .
$$

donde: $M$ es mortalidad natural, $L C \infty$ la talla asintótica de cefalotórax, $K$ el coeficiente de crecimiento y $T=$ temperatura promedio del mar en ${ }^{\circ} \mathrm{C}$.

También se calculó el valor de $M$ con la fórmula de Rikhter \& Efanov (1976) utilizando FISAT (Gayanillo Jr. et al. 2005):

$$
M=((1.52 / \text { tmass }) \cdot 0.72)-0.16
$$

donde: $M$ es mortalidad natural y tmass la edad de maduración sexual masiva.

La mortalidad total $Z$ se estimó usando curvas de capturas por talla en la subrutina de FiSAT II (Gayanillo Jr. et al. 2005).

La estimación de la mortalidad por pesca $F$ se realizó por medio de la siguiente fórmula (Sparre \& Venema 1997):

$$
\mathrm{F}=\mathrm{Z}-\mathrm{M}
$$

donde $F$ es tasa instantánea de mortalidad por pesca, $Z$ la tasa instantánea de mortalidad total, $M=$ tasa instantánea de mortalidad natural (Cadima 2003).

El coeficiente de explotación es definido como (Gulland 1971):

$$
\mathrm{E}=\mathrm{F} / \mathrm{Z}
$$


donde: $E=$ tasa de explotación, $F=$ tasa de mortalidad por pesca, $Z=$ tasa instantánea de mortalidad total (Cadima 2003).

El criterio empleado para determinar el grado de explotación del stock, se definió de acuerdo al criterio de Gulland \& Carroz (1968):

$\mathrm{E}=0.5$, la población está en el punto óptimo de explotación

$\mathrm{E}<0.5$, la población esta sub-explotada

E $>0.5$, la población esta sobreexplotada

Con el programa de evaluaciones pesqueras FiSAT II, se obtuvieron los patrones de reclutamiento. Dicho programa utiliza los datos de frecuencias de tallas para identificar el número, localización y magnitud de los picos de reclutamiento. Al aplicar este análisis se debe tener cautela, ya que el modelo está basado en dos supuestos que raramente se encuentran en la realidad biológica de la especie bajo estudio: a) todo individuo en la simulación de crecimiento es descrito por un conjunto simple de parámetros de crecimiento, b) hay un mes dentro del año que siempre se computará con un reclutamiento de 0 (Gayanillo Jr. et al. 2005).

\section{RESULTADOS}

Estructura de tallas: Las 843 langostas analizadas presentaron una longitud cefalotoráxica (LC) promedio de $72.5 \mathrm{~mm}( \pm 15 \mathrm{DE}$, 35.8-143.6). Esta estructura evidencia la presencia de un elevado porcentaje de langostas pequeñas por debajo de la talla mínima legal (80mm de LC) que comprenden el $74.9 \%$ de las capturas (Fig. 1). Estas características estructurales reflejan que la pesca de langosta en esta región esta teniendo lugar muy cerca o sobre las áreas de cría.

La LC promedio de los machos fue de $75.1 \mathrm{~mm}( \pm 16.5 \mathrm{DE}, 42.8-143.6)$. En las

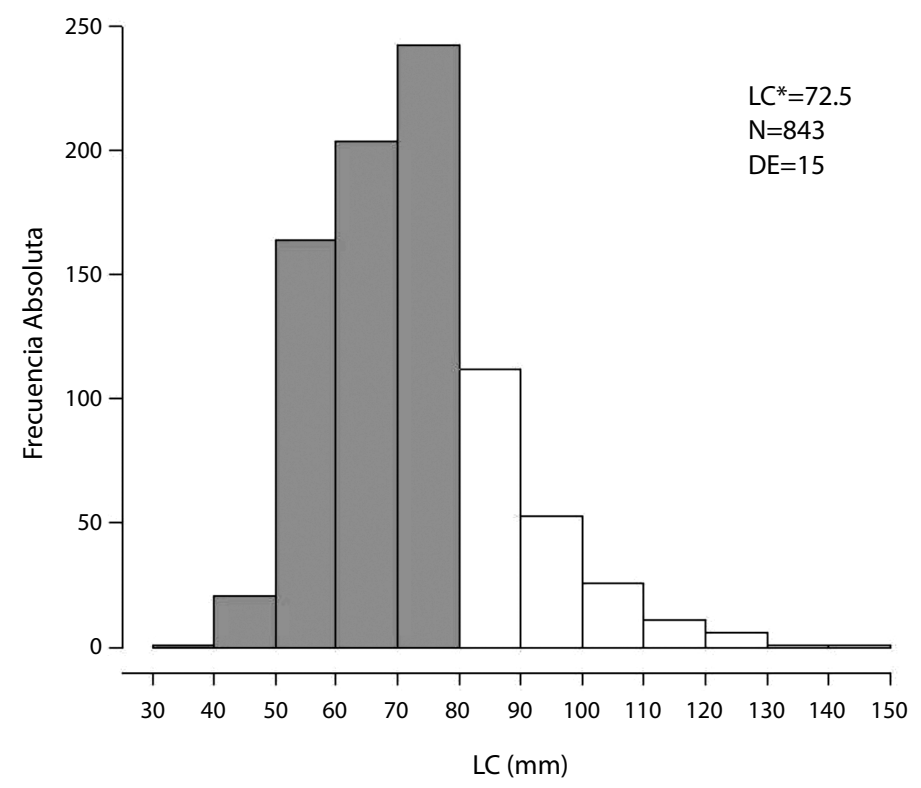

Fig. 1. Estructura de clases de LC en la pesquería de la langosta $P$. gracilis en la zona de Playa Lagarto. Las columnas sombreadas indican ejemplares por debajo de la talla mínima legal de comercialización ( $80 \mathrm{~mm}$ de LC). *=promedio de LC. Fig. 1. LC frequency distributions of $P$. gracilis in Playa Lagarto. The shaded columns indicate samples below the legal minimun size ( $80 \mathrm{~mm}$ of LC). *=mean of LC. 
hembras el promedio fue un poco menor: $69.1 \mathrm{~mm}( \pm 11.8 \mathrm{DE}, 35.8-115)$. La talla promedio de las hembras reproductivas fue superior a la del total de hembras con $74.3 \mathrm{~mm}( \pm 10.9 \mathrm{DE}$, $35.8-115)$ y la menor talla global de madurez registrada fue de $35.8 \mathrm{~mm}$, la cual correspondió a una hembra ovígera o con fresa y sin masa espermatófora. El $67.2 \%$ del total de hembras de la muestra presentó algún atributo de madurez reproductiva con las siguientes características: 49 langostas sin fresa pero con masa espermatófora, 54 con fresa y sin masa espermatófora, y 100 ejemplares con ambas condiciones (Cuadro 1).

Al comparar las tallas entre machos y hembras se encontraron diferencias significativas entre las medias $(\mathrm{t}=-4.99, \mathrm{gl}=356, \mathrm{p}<0.05)$. Además, el $86.6 \%$ del total de hembras y un $69.3 \%$ de machos se encontraban por debajo de la talla mínima de comercialización y estan representados en la figura por las barras sombreadas (Fig. 2).
Relación talla-peso: La relación entre talla de longitud cefalotóraxica y peso revela que las hembras pesan más que los machos de una misma talla y que esta diferencia es significativa $(\mathrm{p}<0.05)$. El peso máximo registrado correspondió a un macho de 1987.3 g con una talla de $143.6 \mathrm{~mm}$, mientras que la hembra de mayor talla pesó $1258.7 \mathrm{~g}$ con una talla de $115 \mathrm{~mm}$. Conforme las tallas van incrementando, la relación de los pesos entre machos y hembras aumenta a favor de éstas últimas. El peso húmedo promedio de las langostas hembras ovígeras o no ovígeras equivalentes a la talla legal $(80 \mathrm{~mm}$ de LC) fue de $434.3 \mathrm{~g}$, mientras que para los machos fue de $400.7 \mathrm{~g}$, o sea que la hembra en ese tamaño pesa un $7.8 \%$ más. Este incremento en peso puede ser atribuido a la presencia de la masa espermatófora y ovígera; además se indicó que en muchas ocasiones la acumulación de agua en estos últimos tejidos incidía en el momento en que éstas eran pesadas (Fig. 3).

\section{CUADRO 1}

Estructura de tallas absolutas por clases de LC ( $\mathrm{mm})$ y frecuencia de machos, hembras y hembras reproductivas en la muestra total de langostas $P$. gracilis. N=Tamaño de muestra

TABLE 1

Size composition of LC (mm) and frequency by sex in the total sampled of $P$. gracilis. N=sample size

$\begin{array}{ccccc}\text { Clases de LC } & \mathrm{N} & \text { Machos } & \text { Hembras } & \text { Hembras reproductivas } \\ 30-40 & 1 & 0 & 1 & 1 \\ 41-50 & 20 & 8 & 11 & 1 \\ 51-60 & 163 & 86 & 77 & 20 \\ 61-70 & 201 & 106 & 95 & 46 \\ 71-80 & 247 & 137 & 110 & 78 \\ 81-90 & 113 & 71 & 42 & 38 \\ 91-100 & 52 & 33 & 19 & 0 \\ 101-110 & 27 & 27 & 0 & 1 \\ 111-120 & 11 & 10 & 1 & 0 \\ 121-130 & 6 & 6 & 1 & 0 \\ 131-140 & 1 & 1 & 0 & 0 \\ 141-150 & 1 & 1 & 0 & 203 \\ \mathrm{~N} & 843 & 486 & 357 & 74.3( \pm 10.9 \mathrm{DE}) \\ \text { Promedio } & 72.5( \pm 15 \mathrm{DE}) & 75.1( \pm 16.5 \mathrm{DE}) & 69.1( \pm 11.8 \mathrm{DE}) & 115 \\ \text { Máximo } & 143.6 & 143.6 & 115 & 35.8 \\ \text { Mínimo } & 35.8 & 44.2 & 35.8 & \end{array}$



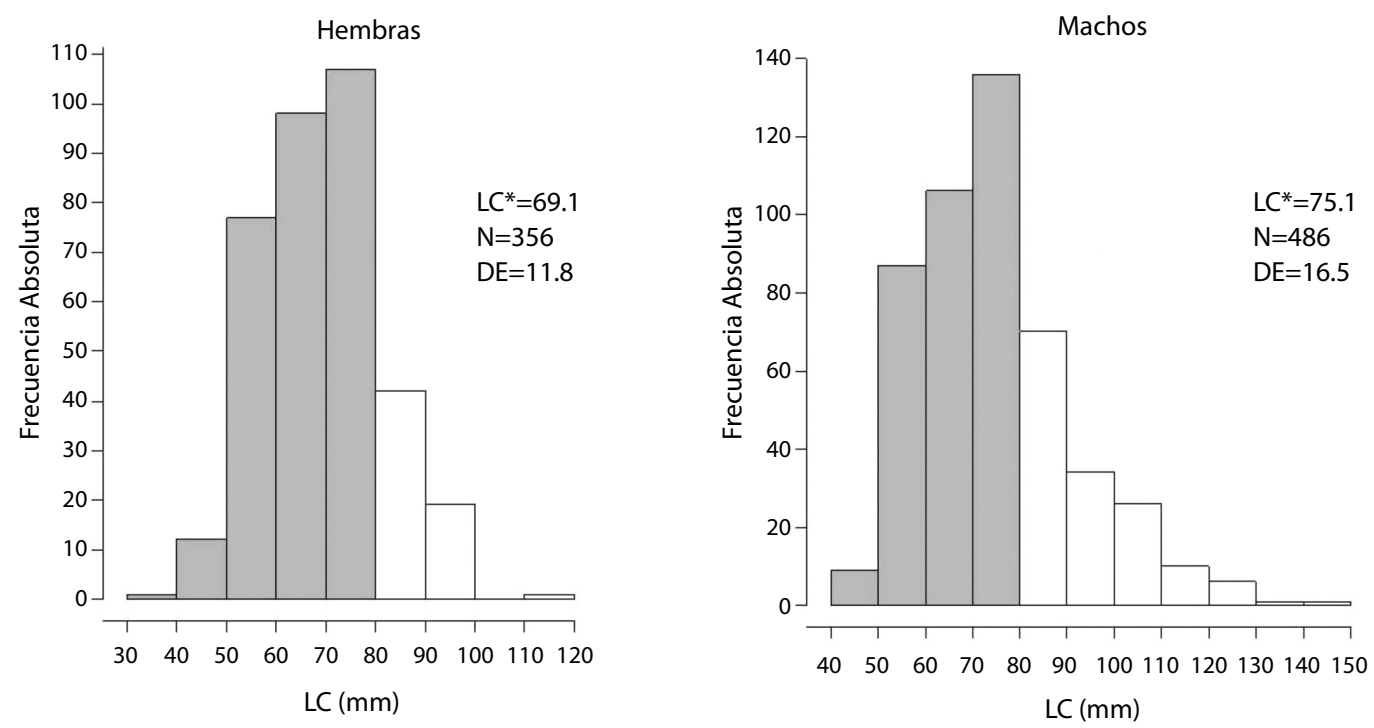

Fig. 2. Distribución de frecuencias absolutas de tallas por sexo en la muestra total de langostas P. gracilis, Playa Lagarto.*=promedio de LC.

Fig. 2. LC frequency distributions by sex of the total sampled of P. gracilis in Playa Lagarto.

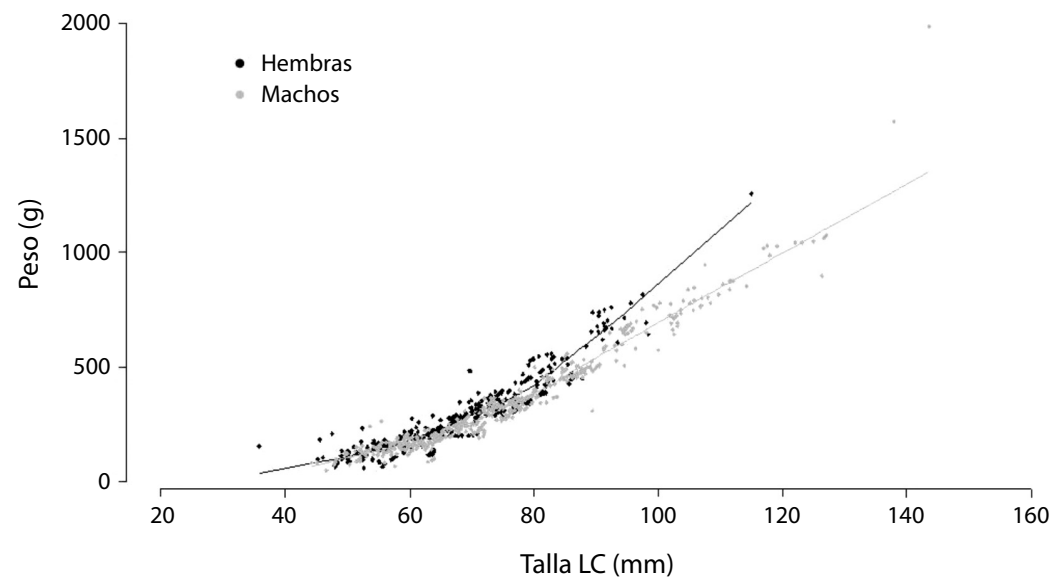

Fig. 3. Relación talla-peso por sexo para la langosta P. gracilis en Playa Lagarto. Se muestra la línea de tendencia para cada sexo.

Fig. 3. Length-weight relationship by sex of P. gracilis in Playa Lagarto. It shows the tendency line for each sex.

Por las razones mencionadas anteriormente, se procedió a agrupar las hembras no ovígeras para establecer la relación talla-peso en comparación con el total de machos. Las diferencias entre las medias entre peso y talla por sexo fue significativa $(\mathrm{p}<0.05)$ a favor de las hembras. De esta forma, siguió existiendo un ligero aumento en las hembras principalmente partir de tallas mayores a los $80 \mathrm{~mm}$ de LC, que podría deberse a que éstas últimas presentan 
más volumen y superficie en la región abdominal para acomodar la masa de huevos.

El modelo de regresión entre las variables longitud de cefalotórax-peso explica un mejor ajuste para los machos $(y=0.509+0.069 *$ Peso $)$ que para las hembras $\left(y=0.489+0.006 *\right.$ Peso), con valores de $r^{2}$ igual a 0.91 y 0.86 respectivamente:

Distribución de los sexos: Se encontraron diferencias significativas en la proporción anual de machos y hembras $\left(\mathrm{X}^{2}=19.74, \mathrm{gl}=1\right)$. El porcentaje de machos fue $1.36 \mathrm{M}: \mathrm{H}$.
En el intervalo de distribución de las cajas se evidencia la presencia de un alto solapamiento, y la tendencia al incremento de tallas mayores con relación a la profundidad. La mezcla de los segmentos poblacionales refleja una condición ecológica natural de la necesidad de estos organismos en residir temporalmente en diferentes tipos de hábitats bentónicos según requerimientos ontogéneticos, migratorios, gregarios, alimentarios y reproductivos antes de llegar a ocupar estratos más profundos.

Lo anterior revela que ambos métodos de captura estarían impactando sobre posibles

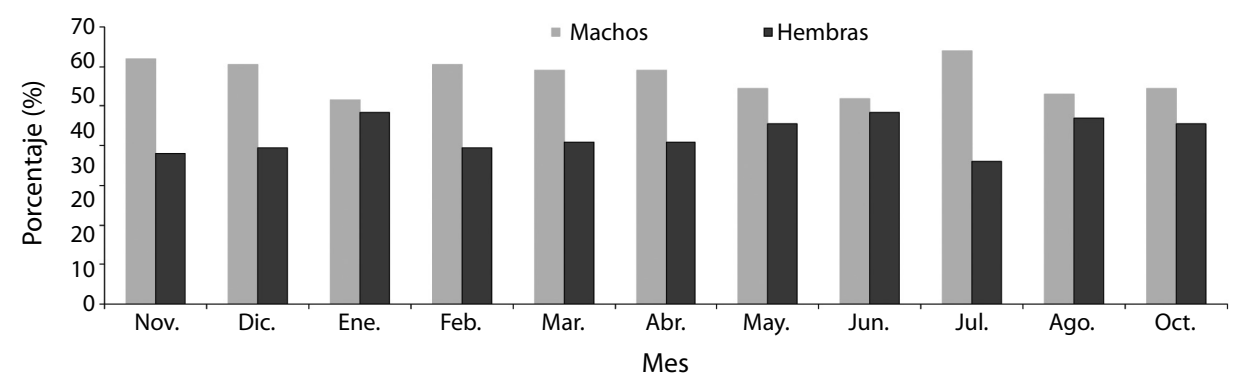

Fig. 4. Porcentaje mensual de machos y hembras de P. gracilis en la zona arrecifal de Playa Lagarto.

Fig. 4. Sex proportion of $P$. gracilis by month in Playa Lagarto.

La distribución de sexos por mes evidencia que los machos fueron dominantes durante todo el año, superando el $50 \%$ durante todos los meses. A julio y noviembre les correspodió las mayores abundancias con más del $70 \%$ del total. Las hembras por su parte, presentaron mayores porcentajes en enero, mayo, junio, agosto y octubre (Fig. 4).

Estructura de tallas y variaciones del sexo con la profundidad: Al comparar la distribución por sexo y por tallas de $P$. gracilils de las muestras del buceo con compresor y pulmón, relacionado con las diferencias en la profundidad, encontramos algunas divergencias.

En la Fig. 5 se muestra la distribución de las tallas del cefalotórax, en al comparación con los estratos somero del buceo a pulmón (1 a 5 brazadas) y el estrato profundo correspondiente al buceo con compresor (6 a 18 brazadas).

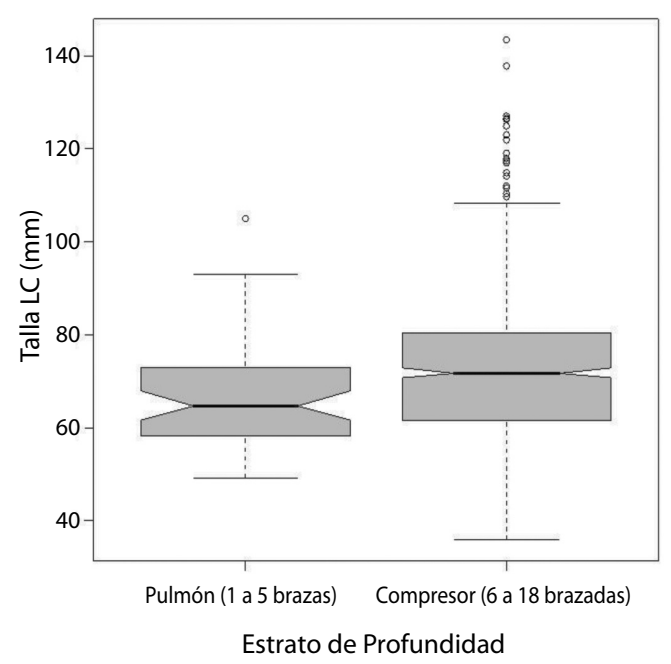

Fig. 5. Gráfico de caja que muestra la distribución de tallas de LC de $P$. gracilis en los estratos de profundidad en brazadas por método de pesca.

Fig. 5. Box plot of CL size distribution of P. gracilis in the layers of depth (fathom) by fishing method. 
zonas de cría y/o post asentamiento de langostas en estado juveniles o inmaduras. La talla media de LC del segmento arrecifal profundo registro un valor de $72.9( \pm 15.1 \mathrm{DE})$ y el de la zona somera un valor de $66.7( \pm 11.5 \mathrm{DE})$. Además, se encontraron diferencias significativas entre las medias $(\mathrm{p}<0.05)$. Nuevamente, se observa el predominio de langostas sublegales en las capturas que en el estrato de menor profundidad alcanzan el $87.7 \%$, mientras que en las zonas más profundas se reducen a un $74 \%$.

La proporción de sexos entre hembras y machos en las áreas someras exhibió un menor porcentaje para las hembras con un $49.1 \%$ y para los machos un $50.8 \%\left(\mathrm{X}^{2}=0.02, \mathrm{gl}=1\right)$. En las áreas profundas se encontró una variación

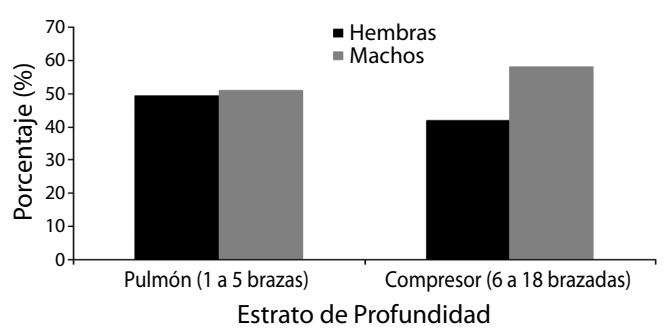

Fig. 6. Porcentaje de variación sexual por estrato de profundidad de la langosta $P$. gracilis.

Fig. 6. Sex proportion variation by layer of depth of P. gracilis. mayor de $41.8 \%$ hembras y $58.1 \%$ machos $\left(\mathrm{X}^{2}=20.8, \mathrm{gl}=1\right)$ (Fig. 6).

Parámetros de crecimiento: Los parámetros de crecimiento fueron calculados de manera independiente para cada sexo. Así, la longitud cefalotoráxica asintótica $(L C \infty)$ fue estimada en $166.9 \mathrm{~mm}$ (IC $95 \%=165.4 \rightarrow$ 167.8 ) y $121.7 \mathrm{~mm}$ (IC $95 \%=120.3 \rightarrow 122.9$ ), para machos y hembras respectivamente. Se obtuvo el mejor ajuste para cada caso, con un $\mathrm{r}^{2}$ para las hembras de 0.89 y para los machos de 0.95 .

La tasa de crecimiento o parámetro de curvatura $(K)$ mostró valores relativamente altos en comparación con estimaciones para otras especies de Panulirus. Las puntuaciones ("S") fueron de 0.992 y 0.971 para machos y hembras, respectivamente.

Adicionalmente, se estimaron los índices de crecimiento ( $\varnothing^{\prime}$ primo) para machos y hembras de 2.090 y 1.7. Esto con el fin de examinar diferencias entre el presente estudio anual y otros parámetros de crecimiento en otras áreas, períodos y/o especies (Cuadro 2).

Según estas estimaciones, las hembras alcanzarían la talla de madurez sexual a una edad de 2.1 años y la talla mínima de captura oficial (80mm de LC) a una edad de 2.9 años. Los machos alcanzan una $L C_{\infty}$ mucho más alta

CUADRO 2

Mortalidad total $(\mathrm{Z})$, mortalidad natural $(\mathrm{M})$ y mortalidad por pesca $(\mathrm{F})$ para machos y hembras con respecto al método utilizado. (*) La mortalidad natural fue estimada con el método de Cruz et al. (1981)

TABLE 2

Total mortality (Z), natural mortality (M) and fishing mortality (F) for males and females $(*)$ Natural mortality was estimated using Cruz et al. (1981) method

\begin{tabular}{|c|c|c|c|c|}
\hline Mortalidad & Método & Sexo & Valor & $\begin{array}{l}\text { Otros Estudios } \\
\text { Hearn et al } 2008\end{array}$ \\
\hline$Z$ & Curva de Captura & Machos & 2.34 & 0.79 \\
\hline$Z$ & Curva de Captura & Hembras & 1.82 & 0.68 \\
\hline M & Cruz et al. & Machos & 0.49 & 0.34 \\
\hline$M$ & Cruz et al. & Hembras & 0.47 & 0.36 \\
\hline$M$ & Rickter \& Efanov & Machos & 1.3 & 0.42 \\
\hline$M$ & Rickter \& Efanov & Hembras & 0.9 & 0.42 \\
\hline$F$ & $\mathrm{~F}=\mathrm{Z}-\mathrm{M}^{*}$ & Machos & 1.92 & - \\
\hline$F$ & $\mathrm{~F}=\mathrm{Z}-\mathrm{M}^{*}$ & Hembras & 1.42 & - \\
\hline
\end{tabular}


que las hembras y exhiben también una tasa de crecimiento mayor por lo que alcanzan la talla mínima de captura legal a la edad de 1.4 años, lo que representa una diferencia pronunciada de crecimiento de 1.5 años entre ambos sexos (Cuadro 3). En la Fig. 7 se detalla la curva de crecimiento por talla de LC con base a la ecuación de von Bertalanffy.
Mortalidad y patrones de reclutamiento: Las estimaciones en la mortalidad natural variaron considerablemente según el método empleado. Con el método de Cruz et al. (1981) se observa una mortalidad muy parecida de 0.41 como promedio para machos y hembras con una temperatura promedio anual de $23^{\circ} \mathrm{C}$. Los resultados del método Rickter \& Efanov

CUADRO 3

Comparación de los parámetros de crecimiento por sexo y método de P. gracilis con respecto a otras especies

TABLE 3

$P$. gracilis growth parameters by sex and method compared to other species

\begin{tabular}{lcccccc}
\multicolumn{1}{c}{ Método } & Sexo & $\begin{array}{c}\text { Parámetro de } \\
\text { Crecimiento }\end{array}$ & $\begin{array}{c}\text { Este } \\
\text { estudio }\end{array}$ & Hearn et al. 2008 & Barnutty 2000 & Sosa-Cordero 1997 \\
Powell-Wetherall & Machos & $L C_{\infty}$ & 166.9 & 169.5 & 269 & 223 \\
Powell-Wetherall & Hembras & $L C_{\infty}$ & 121.7 & 123.8 & 261 & 216 \\
Sheperd & Machos & $K$ & 0.45 & 0.2 & 0.22 & 0.29 \\
Sheperd & Hembras & $K$ & 0.38 & 0.26 & 0.2 & 0.28 \\
Rutina FiSAT & Machos & $\varnothing^{\prime}$ & 2.1 & 1.48 & 4.21 & - \\
Rutina FiSAT & Hembras & $\emptyset^{\prime}$ & 1.7 & 1.95 & 4.14 & -
\end{tabular}

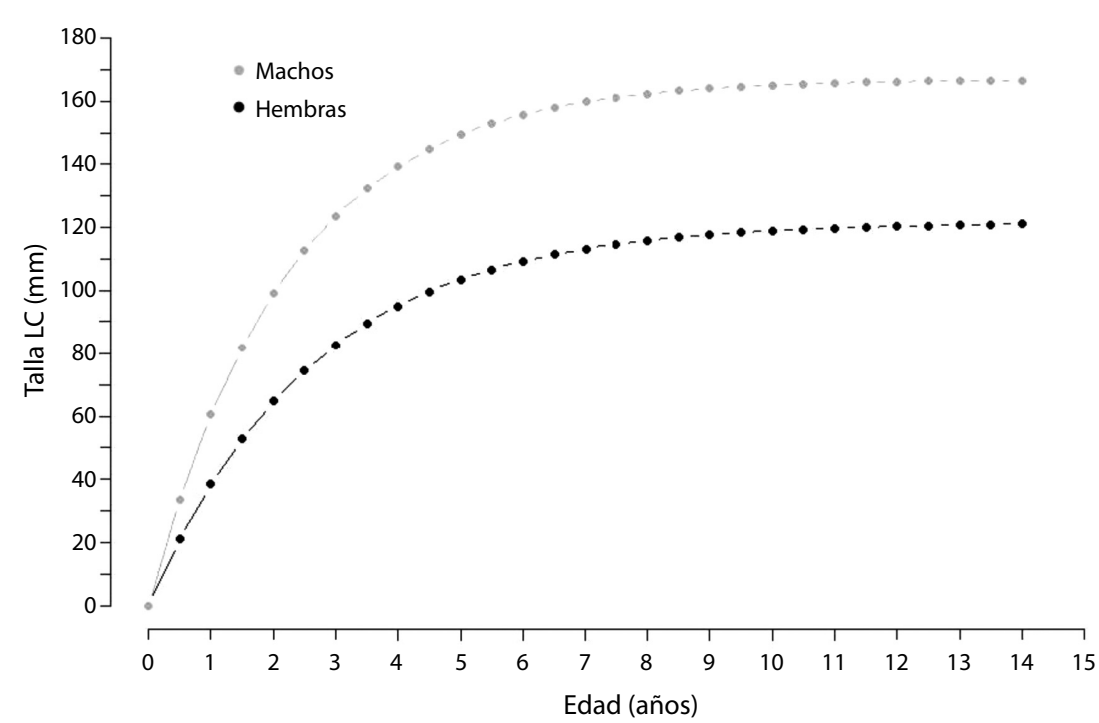

Fig. 7. Curvas de crecimiento por talla de LC $(\mathrm{mm})$ con respecto a la edad (años) para machos y hembras de la langosta $P$. gracilis.

Fig. 7. Growth curves of LC size (mm) in response to age (years) for both sex of P. gracilis. 
(1976) son un poco más altos con un 1.1 como promedio para los dos sexos. Sin embargo, ambos métodos suponen una mayor mortalidad para los machos.

Los estimados para la mortalidad total $(Z)$ indican una considerable diferencia entre machos y hembras con 2.34 y 1.82 , respectivamente. Estas altas tasas de mortalidad total sin duda se encuentran asociadas a las también altas tasas de mortalidad por pesca de 1.92 para machos y 1.42 para hembras (Cuadro 3).

Para la tasa de explotación $(E)$ se obtuvo un valor de 0.80 , lo cual evidencia que la población se encuentra bajo una condición de intensa explotación pesquera de acuerdo con el criterio establecido previamente.

El reclutamiento para ambos sexos presentó un comportamiento continuo durante el año con dos pulsos de diferente intensidad, el primero en Febrero, con un 5\% y el segundo de mayor intensidad en Julio con un 18.5\% (Fig. 8).

\section{DISCUSIÓN}

Las características estructurales observadas durante el año de muestreo sobre una porción explotable de la población de langostas en
Playa Lagarto se distribuyeron en un amplio rango de tallas. Las mismas comprenden desde 42.8 a $143.6 \mathrm{~mm}$ de LC para los machos y 35.8 a $115 \mathrm{~mm}$ de LC para las hembras. Existe una tendencia clara de aumento de tallas con la profundidad.

Además, gran parte del esfuerzo pesquero está afectando una importante proporción del segmento juvenil y esto podría estar provocando un fenómeno conocido como sobre-pesca de crecimiento lo que afecta el potencial reproductivo al extraer langostas que no han participado en la reproducción (Sosa-Cordero et al. 1993). Así mismo, existe poca disponibilidad de tallas legales o superiores a $80 \mathrm{~mm}$ de LC que deberían sustentar a la pesquería en condiciones de un manejo sostenible.

El hecho de encontrar bajos porcentajes de hembras de tallas mayores a $100 \mathrm{~mm}$ de LC podría estar relacionado con varios factores: 1) El gradiente de profundidad que limita al buzo a no bajar a más de $40 \mathrm{~m}$, asociado al hecho de que las hembras en el momento del desove tienden a buscar zonas profundas (Lyons et al. 1981) más allá de esta isóbata. 2) No se descarta que puedan existir agregaciones o parches poblacionales en zonas arrecifales por

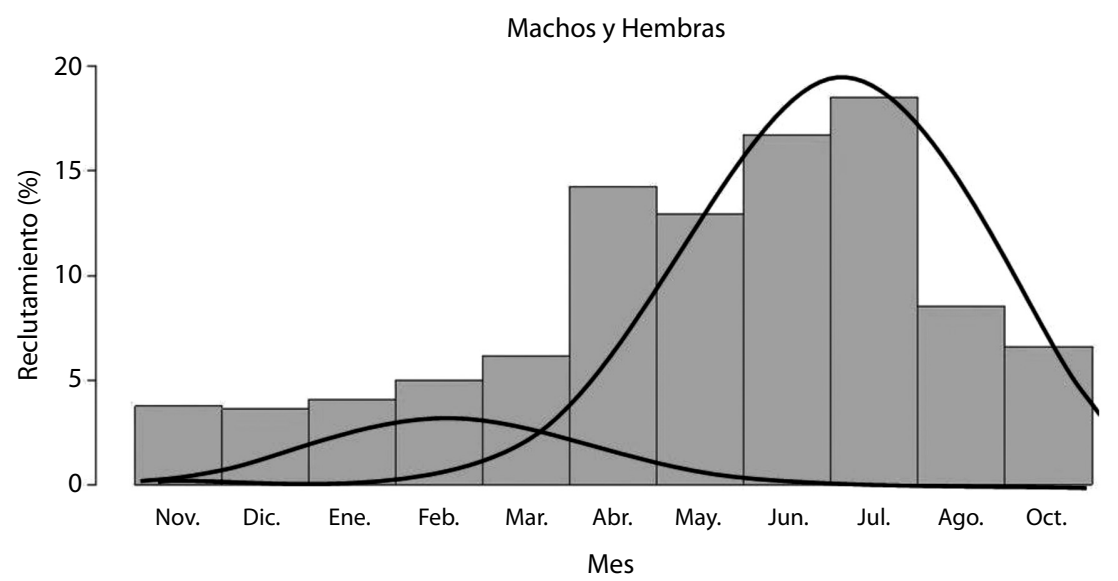

Fig. 8. Patrón de reclutamiento anual de P. gracilis. Las líneas negras indican los mayores pulsos que se dieron en febrero (5\%) y Julio (18.5\%).

Fig. 8. Annual recruitment pattern of P. gracilis. The black lines indicate the major pulses that occurred in February (5\%) and July $(18.5 \%)$. 
debajo de los 40m, en las cuales no faena la flota con compresor y donde se podrían encontrar individuos de mayores tallas. 3) La fuerte presión de pesca que existe sobre el segmento poblacional juvenil que remueve gran parte de la estructura poblacional de éstas tallas, por lo cual disminuye la abundancia y disponibilidad de tallas adultas (sobre-pesca en el reclutamiento). Esto es válido tanto para hembras como para machos.

En lo referente a la proporción sexual se determinó que a través del año siempre hubo predominancia en los machos (57.6\%). Este valor es levemente inferior al encontrado por Guzmán (2008) en las costas panameñas quién determinó un $70 \%$ de dominancia de los machos en las capturas de la pesca comercial y pescas exploratorias en los archipiélagos de Las Perlas y Coiba. En general, este contraste puede ser causado por diferentes conductas relacionadas con la madurez sexual que provocan que los intervalos por sexo varíen con respecto a los estratos de profundidad. Al respecto, Estrella \& McKiernan (1989) señalan que éstas diferencias encontradas entre la proporción de sexos en el estrato profundo, pudieran estar fundamentadas en la hipótesis de que los machos maduros y adultos compiten por los territorios o madrigueras donde ocurren los apareamientos.

La relación entre talla y peso muestra una tendencia al aumento en peso de las hembras que puede apreciarse en las tallas mayores a los 80mm de LC. En contraste, los machos de $P$. penicillatus en las Islas Galápagos registraron más peso que las hembras a partir de tallas mayores a los $21 \mathrm{~cm}$ de longitud total (Hearn 2004).

Las diferencias de LC entre machos y hembras podrían deberse a que los machos invierten menos energía en la producción de gametos que las hembras en la producción de huevos lo que les permite alcanzar a los primeros mayores tallas (Herrkind \& Lipcus 1989).

Vale mencionar que aún cuando existe una medida vigente de protección para las hembras ovígeras, el resultado de las proporciones entre machos y hembras no se encontró condicionado por ésta, ya que en esta zona dicha regulación no es implementada y las langostas son capturadas por los pescadores independientemente del estado reproductivo en que se encuentren. Además, el efecto que tendría esta incidencia sobre los individuos reproductores locales es que si éstos generan al menos parte de los reclutas locales, su pesca excesiva reduciría el número de futuros reproductores lo que se conoce como sobre-pesca del reclutamiento (Sosa-Cordero et al. 1993).

En los datos de crecimiento obtenidos con el programa FiSAT II se puede observar un crecimiento relativamente rápido consistente con la estructura de tallas registrada durante el muestreo. Los altos valores de $K$ en contraste a los valores encontrados en otras especies del mismo género, indican una tasa de crecimiento acelerado para $P$. gracilis en esta región. Este ritmo de crecimiento de cierta forma podría explicar las observaciones asociadas con la temprana talla de madurez sexual encontrada (Naranjo 2009). El macho llega ser más grande que la hembra, lo cual coincide con la mayor proporción de machos de tallas grandes registradas y con la mayoría de cálculos de $L^{\infty}$ en otros estudios. Además, la variabilidad en los niveles de crecimiento en ambos sexos es parecida con las encontradas en poblaciones de otras especies (Morgan 1980).

Hay que tener en cuenta que la construcción de las curvas de crecimiento no se basó en la estructura de los estados de muda, lo que no es muy representativo para el crecimiento escalonado que presentan los crustáceos. Por lo que se recomienda incorporar el ajuste ponderado teniendo en consideración los grupos modales correspondientes (Zetina-Moguel \& Ríos-Lara 2000). Sin embargo, aún cuando no se incorporó un modelo de ajuste escalonado, se aprecia concordancia de las edades obtenidas en este estudio y otros, teniendo presente las posibles variables implícitas en los parámetros de crecimiento de diferentes especies para distintos lugares. Por ejemplo, la longitud de cefalotórax de $P$. argus en México (Zetina-Moguel \& Ríos-Lara 2000) a una edad de 2.4 años fue de $61.8 \mathrm{~mm}$ de LC, mediante el uso de un modelo 
escalonado. Mientras que una langosta $P$. gracilis de esa misma talla tendría una edad de 1.9 años, o sea esto constituiría una diferencia de aproximadamente 5 meses.

Para la comparación de individuos de la misma especie (Pauly 1984) con otras, se recurrió al uso del índice de crecimiento intraespecífico $\left(\emptyset^{\prime}\right)$, debido a falta de estudios donde se calculen los parámetros de crecimiento para $P$. gracilis. Se encontró que los valores son relativamente aceptables y parecidos a los estimados de P. penicillatus en las Islas Galápagos donde se uso la longitud asintótica del cefalotórax para el cálculo de $\varnothing^{\prime}$ al igual que en ésta investigación. El valor de $\varnothing^{\prime}$ de $P$. argus (Barnutty 2000) fue estimado con $L \infty$ de la longitud total por lo cual muestra valores altos, muy superiores a los obtenidos para $P$. gracilis.

Los estimados de los parámetros de crecimiento en la presente investigación se basaron exclusivamente en el análisis modal de frecuencias de talla. Sin embargo, se debe tener en cuenta que el estudio de las tallas es generalmente considerado como una medida pobre de la edad en crustáceos que viven en hábitats naturales debido a que el crecimiento es dependiente de muchos factores ambientales y es afectado además por la denso-dependencia (James et al. 2001). Una técnica alternativa para el cálculo de la edad en crustáceos ha sido desarrollada en base a la concentración de un pigmento de edad, llamado neurolipofuscin, el cual se concentra en tejidos suaves del sistema nervioso central y que ha sido utilizado como un buen indicador de edad en muchas especies de crustáceos (Maxwell et al. 2007). Por esta razón, se deben proponer estudios histológicos que permitan corroborar nuestras aproximaciones de edad encontradas para P. gracilis en Playa Lagarto.

Otro aspecto relacionado al crecimiento que hay que tener en cuenta, es que existe un impacto del arte de pesca que se utiliza en esta región sobre los individuos que logran escapar. Durante los desembarques y los muestreos a bordo de las embarcaciones se comprobó la presencia de ciertos ejemplares con ausencia de algún apéndice o lesión. Se ha comprobado que este tipo de manipulación por pesca retarda el crecimiento de un 15 a un 50\% (Waugh 1981, Kuris \& Mager 1975). En algunos casos, langostas con lesiones tienen reducidos lapsos de inter-mudas y cortos ritmos de crecimiento en comparación a langostas sin lesiones (BrionesFourzán \& Lozano 2000). De esta manera, se necesitan estudios que permitan cuantificar la magnitud de este fenómeno y su grado de afectación sobre los parámetros de crecimiento.

Los machos presentan una mayor tasa de mortalidad $(Z)$ que las hembras lo cual es correspondiente con la proporcionalidad encontrada entre machos y hembras durante el año. Además, hembras y machos son afectados por altas tasas de mortalidad por pesca con $77.9 \%$ y $81.9 \%$ respectivamente. Los valores de mortalidad natural fueron muy diferentes según la metodología utilizada. No obstante, se tomó la ecuación desarrollada por Cruz et al. (1981) como base para la estimación de las tasas de mortalidad por pesca, debido a que dicha ecuación fue diseñada particularmente para langostas espinosas. La mortalidad natural $(M)$ de los recursos marinos es uno de los parámetros más difíciles de estimar, los métodos generados para el calcularlo han sido para peces, organismos que tienen una biología diferente a la de los crustáceos. A su vez, gran parte de los métodos bio-analógicos empleados para su estimación dependen de los parámetros de crecimiento de von Bertalanffy, principalmente $K$, por lo que si éstos presentan algún grado de error o imprecisión las estimaciones de $\mathrm{M}$ se ven directamente afectadas (Arana \& Olate 2000).

El pulso de mayor intensidad en el patrón de reclutamiento estimado en base a la estructura de tallas coincidió con el periodo de mayor actividad reproductiva (Naranjo 2009). El primer pulso ocurrió en Febrero con un 5\% y el segundo de mayor intensidad en julio con un $18.5 \%$. El hecho de que esta especie haya presentado una alta actividad reproductiva anual (Naranjo 2009), es consistente con el patrón de reclutamiento el cual se mantuvo con valores superiores al $5 \%$ durante la mayor parte del año. Cabe mencionar que en esta región los individuos se reclutan al arte de pesca a los 25 meses (2.1 años) que corresponde a una talla 
muy baja de $35 \mathrm{~mm}$ de LC y sería la talla de primera captura. Desafortunadamente, en la literatura consultada no se establecen variaciones temporales en el reclutamiento de $P$. gracilis en el Pacífico que permitan relacionar un posible patrón cíclico que concuerde con los pulsos considerados en este estudio.

Además de la talla mínima de captura oficial, en Costa Rica existen otras regulaciones básicas para la captura de langosta como la prohibición de comercialización de hembras portadoras de huevos. No obstante, durante esta investigación se pudo comprobar que dichas regulaciones no se cumplen y esta situación que ha caracterizado la actividad pesquera durante muchos años en esta zona, pudo haber provocado un efecto de sobreexplotación del recurso que es confirmado por la alta tasa de explotación obtenida de 0.80 . Las bajas tallas de madurez sexual encontradas en esta región (Naranjo 2009) y los niveles en los parámetros de crecimiento, pueden constituir una respuesta compensatoria de la población a la fuerte presión de pesca ejercida a través de varias décadas que ha desencadenado una población de jóvenes.

Debido a lo anterior, resulta indispensable que se cumplan a cabalidad las regulaciones existentes relacionadas con el respeto a la talla mínima de captura, la no captura de hembras con huevos y evitar pescar las especies vedadas. Esta medida contribuiría a mediano plazo a incrementar el número de ejemplares de tamaño comercial en el mar además de mejorar el reclutamiento para los años siguientes lo que aumentaría la biomasa del recurso.

Además, se recomienda buscar artes de pesca selectivas que restrinjan la composición de tallas en las capturas, principalmente con el fin de proteger la presión sobre el segmento poblacional juvenil en las áreas someras y la posible incidencia del arte actual sobre los parámetros de crecimiento de la especie.

\section{AGRADECIMIENTOS}

Extiendo un especial agradecimiento a los pescadores de Playa Lagarto, quienes hicieron posible la realización de los estudios llevados a cabo en el área: Luis Calderón, Jeudi Ortiz, Junior Espinoza, Leiter y a todos aquellos que de una u otra forma colaboraron en esta investigación. En la Universidad Nacional agradezco el apoyo de Luis Angel Palacios, Rosa Soto, Luis Villalobos, Manuel Espínola, Luis Adrián Vega, Hannia Vega, Luis Sierra y Rigoberto Víquez. En el CINVESTAV a Silvia Salas por sus oportunas aclaraciones.

\section{RESUMEN}

Los parámetros biológicos y pesqueros analizados se fundamentaron en una muestra de 843 langostas provenientes de la actividad pesquera realizada en Playa Lagarto entre noviembre del 2007 y octubre del 2008. Las longitudes cefalotoráxicas de los machos estuvo comprendida entre los 143.6 y 42.8 y 115 y $35.8 \mathrm{~mm}$ para hembras. El $74.9 \%$ de las langostas en los desembarques estuvo por debajo de la talla mínima legal de captura $(80 \mathrm{~mm}$ de LC). La estructura de tallas muestra un considerable solapamiento de segmentos poblacionales y el incremento de las tallas con la profundidad en donde operan las dos modalidades de pesca estudiadas: el buceo a pulmón y el buceo con compresor. La proporción de sexos obtenida fue de $1.36 \mathrm{M}: \mathrm{H}$. La relación entre LC y peso reveló que las hembras pesan más que los machos de una misma talla y que esta diferencia fue significativa $(\mathrm{p}<0.05)$. Los parámetros de crecimiento de von Bertalanffy para machos y hembras respectivamente $(K=0.45-0.38, L C \infty=166.9$ 121.7) indican un crecimiento acelerado en comparación a otras especies. Los machos por su parte exhibieron una mayor tasa de crecimiento que las hembras. Además, la mortalidad natural $(M)$, la mortalidad total $(Z)$ y la mortalidad por pesca $(F)$ fue más alta en machos (0.49-2.34-1.92) que en hembras (0.47-1.82-1.42). El reclutamiento para ambos sexos presentó un comportamiento continuó durante el año con un pulso de mayor intensidad en julio de $18.5 \%$. El significativo porcentaje de langostas por debajo de la talla mínima legal permitida para la pesca evidencia fuerte presión ejercida sobre las tallas juveniles. Afirmación que es reforzada por el valor de la tasa de explotación $(E)$ determinada de 0.80 , que indica que bajo el actual régimen de pesca la población estaría en riesgo de colapso.

Palabras clave: Panulirus gracilis, pesquería, Playa Lagarto, Costa Rica.

\section{REFERENCIAS}

Arana, P. \& C. Olate. 2000. Composición de las capturas de la langosta de Juan Fernández (Jasus frontalis) y determinación de los parámetros biológico-pesqueros 
durante la temporada de pesca 1996-1997. Escuela de Ciencias del Mar, Universidad Católica de Valparaíso. Invest. Mar. 28: 83-115.

Barnutty, R. 2000. Aplicación de un modelo de análisis de cohortes a la pesquería de langosta espinosa Panulirus argus del Mar Caribe de Nicaragua. Inf. Tec. CIPA, Managua, Nicaragua.

Beverton, R.J.H. \& S.J. Holt. 1956. A review of methods for estimating mortality rates and fish populations, with special references to sources of bias in catch sampling. Rapp. P. - V. Réun. Cons. Int. Explor. Mer. 140: 67-83.

Briones-Fourzán, P. \& D.E. Lozano. 1977. Aspectos generales sobre la Biología y Pesquería de las langostas (Panulirus inflatus y P. gracilis) en Zihuatanejo, Guerrero y áreas circunvecinas. Tesis Grado, Universidad Nacional Autónoma de México, México D.F., México.

Briones-Fourzan, P. \& E. Lozano. 2000. Factors affecting growth of the spiny lobster Panulirus gracilis and Panulirus inflatus (Decapoda: Palinuridae) in Guerrero, México. Rev. Biol. Trop. 51: 165-174.

Caddy, J.F. 1987. Size-frequency analysis for crustacea: molt increment and frequency models for stock assessment. Kuwait Bull. Mar. Sci. 39: 43-61.

Cadima, E.L. 2003. Manual de evaluación de recursos pesqueros. Documento Técnico de Pesca. No. 393. FAO, Roma, Italia.

Cruz, R., A.T, Ramírez \& R. Coyula. 1981. Crecimiento y Mortalidad de la Langosta Espinosa (Panulirus argus) en la plataforma suroccidental de Cuba. Rev. Cub. Invest. Pesq. 46: 89-119.

Cruz, R. 2002. Manual de Métodos de Muestreo de la Langosta Espinosa. FAO Documento Técnico de Pesca. No. 399. Roma. Italia. 43: 1-15.

Defeo, O., F. Arregín-Sánchez \& F. Sánchez. 1992. Growth study of yellow clam Mesodesma mactroides: A comparative analysis of three length-based methods. Sci. Mar. 56: 53-59.

Gayanillo Jr. F.C., P. Sparre \& D. Pauly. 2005. FAOICLARM Stock Assessment Tools II (FiSAT II). User's guide. FAO Computerized Information Series (Fisheries). No. 8, Revised version. FAO, Roma, Italia.

Gulland, J.A. \& J.E. Carroz. 1968. Management of Fishery Resources. Adv. Mar. Bio. 86: 1-71.
Guzmán, H.M., R. Cipriani, A.J. Vega, M. López \& J.M. Meir. 2008. Population Assessment of the Pacific Green Spiny Lobster (Panulirus gracilis) in the Pacific of Panama. J. Shellfish. Res. 43: 14-23.

Estrella, T.B \& J.D McKiernan. 1989. Catch-per-uniteffort and Biological Parameters from Massachusetts Coastal Lobster (Homarus americanus) Resource: Description and Trends. NOAA Technical Report NMFS 81. NOAA/National Marine Fisheries Service, EEUU.

Hearn, A. 2004. Evaluación de las poblaciones de langostas en la reserva marina de Galápagos. Informe Final 2002-2004. Santa Cruz, Galápagos, Panamá.

Hearn, A. \& C.J. Murillo. 2008. Life History of the Red Spiny Lobster, Panulirus penicillatus, in the Galápagos Marine Reserve, Ecuador. Pac. Sci. 62: 191-204.

Herrkind, W.F. \& R. Lipcius. 1989. Habitat use and population biology of Bahamian spiny lobster. Proc. Gulf. Caribb. Fish. Inst. 39: 265-278.

Holthuis, L.B. 1991. Marine lobsters of the world: an annotated and illustrated catalogue of species of interest to fisheries known to date. FAO, Roma, Italia.

INCOPESCA, 2001. Informe de Labores 2000 - 2001. San José. Instituto Costarricense de Pesca y Acuacultura, San José, Costa Rica.

James, P.J., J.T. Lennard \& M.P. Paewai. 2001. Effect of stocking density and shelter on growth and mortality of early juvenile Jasus edwardsii held in captivity. Mar. Freshw. Res. 52: 1413-1417.

Kuris, A. \& M. Mager. 1975. Effect of limb regeneration on size increase at moult of the shore crab Hemigrapsus oregonensis and Pachygrapsus crassipes. J. Exp. Zool. 193: 353-360.

Lyons, W.G., D.G. Barber, S.M. Foster, F.S. Kennedy Jr \& G.R. Milano. 1981. The Spiny Lobster, Panulirus argus, in the middle and upper Florida Keys: population structure, seasonal dynamics, and reproduction. Florida. Mar. Res. Publ. 38: 1-45.

Maxwell, K.E., T.R. Matthews, M.R. Sheehy, R.D. Bertelsen \& C.H.D Derby. 2007. Neurolipofuscin is a measure of age in Panulirus argus, the Caribbean spiny lobster, in Florida. Biol. Bul. 213: 55-66.

Morgan, G.R. 1980. Population dynamics of Spiny Lobster, p. 189-217. In J.S. Cobb \& B.F. Phillips (eds.). The Biology and Management of Lobsters. Academic, Nueva York, EEUU. 
Munro, J.L. 1982. Estimation of the parameters of the von Bertalanffy growth equation from recapture data at variable time intervals. J. Cons. CIEM. 40: 199-200.

Naranjo, M.H. 2009. Análisis de la pesquería de la langosta Panulirus gracilis (Streets, 1871) en la zona de Santa Cruz, Guanacaste, Costa Rica. Tesis de Maestría, Universidad Nacional, Heredia, Costa Rica.

Pauly, D. 1979. Theory and management of tropical multispecies stocks: A review with emphasis on the Southeast Asian demersal fisheries. ICLARM Stud. Rev. 156: 47-78.

Pauly, D. 1984. Fish population dynamics in tropical waters: a manual for use with programmable calculators. ICLARM Stud. Rev. 42: 297-325.

Richter, V.A. \& V.N. Efanov. 1976. On one of the approaches to estimation of natural mortality of fish populations. ICNAF Res. Doc. 76/IV/8: 1-12.

Sosa-Cordero, E. 1997. Evaluación del Recurso Langosta Panulirus argus en el Banco de Campeche: Análisis Inicial y Programa de Investigación. Informe Final. Consultoría Nacional, Quintana Roo, México.
Sosa-Cordero, E., A. Medina-Queja, A. Ramírez-González, M. Domínguez-Viveros \& W. Aguilar Dávila. 1993. Invertebrados Marinos Explotados en Quintana Roo, p. 709-734. In S.I. Salazar-Vallejo \& N.E. González (eds.). Biodiversidad Marina y Costera de México. Com. Nal. Biodiversidad y CIQRO, México.

Sparre, P. \& S.C. Venema. 1997. Introducción a la Evaluación de Recursos Pesqueros Tropicales, Parte I. Manual. Documento Técnico de Pesca. N ${ }^{\circ} 306$. FAO, Roma, Italia.

von Bertalanffy, L. 1934. Untersuchungen uber die Gesetzlichkeiten des Wachstums 1. Allgemeine Grundlagen der Theorie. Roux Arch. Entwicklungsmech. Org. 131: 613-653.

Waugh, G.H. 1981. Management of juvenile spiny lobster (Panulirus argus) based on estimated biological parameters from Grand Bahama Island Bahamas. Proc. Gulf Caribb. Fish. Inst. 33: 271-289.

Zetina-Moguel, C.F. \& G.V. Ríos-Lara. 2000. Modelos de crecimiento de langosta (Panulirus argus) y un método para calcular edad. Ciencia Pesquera 14: 57-61. 